V. V. Avdeichikov, V. N. Buldakovskil, A. V. Bychkov,

A. S. Vodop'yanov, I. Voitkovska, V. M. Golovatyuk, V. P. Grigor'yev, Z. Guzik, V. P. Zabolotyn, N. I. Zimin, I. B. Issinskii, R. B. Kadyrov, B. K. Kuryatnikov, L. G. Makarov, E. A. Matyushevski1, V. A. Monchinski, T. S. Nigmanov, S. A. Novikov, V. G. Perfeev, V. D. Ryabtsov, A. B. Sadovskii, V. G. Timofeev, I. A. Tyapkin, N. A. Filatova, E. N. Tsyganov, M. D. Shafranov, D. I. Sherstyanov, and D. Yavorska

Joint Institute for Nuclear Research Dubna, USSR

January 1984

(Translated in February of 1986) 


\section{Accelerated Beam Extraction by Means of a Bent Single Crystal at the JINR Synchrophasotron.*}

by V. V. Avdeichikov, V. N. Buldakovskii, A. V. Bychkov, A. S. Vodop'yanov, I. Voitkovska, V. M. Golovatyuk, V. P. Grigor'yev, Z. Guzik, V. P. Zabolotyn, N. I. Zimin, I. B. Issinskii, R. B. Kadyrov, B. K. Kuryatnikov, L. G. Makarov, E. A. Matyushevskit, V. A. Monchinskit, T. S. Nigmanov, S. A. Novikov, V. G. Perfeev, V. D. Ryabtsov, A. B. Sadovskii, V. G. Timofeev, I. A. Tyapkin, N. A. Filatova, E. N. Tsyganov, M. D. Shafranov, D. I. Sherstyanov, and D. Yavorska.

The possibility of the deflection of accelerated particle beams passing through bent single crystals using the channeling process was theoretically predicted by Tsyganov in 1976.1 This phenomenon was observed for the first time in an experiment with the proton beam extracted from JINR synchrophasotron at $8.4 \mathrm{GeV} .{ }^{2}$ The existence of this phenomenon was later confirmed at the CERN , ${ }^{3}$ Leningrad, 4 and Fermilab ${ }^{5}$ accelerators.

Potential applications for the phenomena of charged particles deflection by means of a bent single crystal were discussed by Koshkarev ${ }^{6}$ and Carrigan. ${ }^{7}$ In particular, the use of a bent single crystal for extraction of particle beams from accelerators was suggested by Koshkarev. ${ }^{6}$

In this paper we report proton beam extraction from the JINR synchrophasotron by means of a bent single crystal.

The proton beam was extracted into an existing channel located on the outside of the accelerator ring in the region of the second straight section. To ensure the capture of particles in the channel it was necessary to deflect charged protons from the region of the first straight section by an angle of approximately $35 \mathrm{mrad}$. This deflection was performed by the bent crystal.

As a deflector we used a plate of silicon single crystal measuring $11 \times 10 \times 0.4 \mathrm{~mm}^{3}$ cut in such a way that the crystallographic plane (111) was parallel to the large side of the crystal with an error in angle not exceeding 15 minutes. To ensure control of the bending and initial positioning of the crystal, the external large side of the crystal was polished to a mirror surface to reflect a laser beam. The silicon plate was glued with epoxy resin to the holder, which had a cylindrical frontal surface. The holder, made of duraluminum, was mounted on a goniometer, which was attached to the rod of a stationary target. The goniometer provided a mechanism for turning the crystal around a vertical axis. The goniometer was remotely controlled. The minimum angular step was approximately 40 microradians. The deflector was placed in a fixed position at various distances from the wall of the accelerator vacuum chamber. Alming of the accelerated protons onto the detector was accomplished by decreasing the equilibrium radius of the internal beam.

* Translated by A. Lubinsky and R. Carrigan from "Communication of the Joint Institute for Nuclear Research, Dubna, 1984, \#1-84." 
After being bent by the crystal deflector, the beam traveled in the required direction down the existing channel after passing through the magnetic field of one quadrant of the accelerator. Extraction was performed for three values of the accelerator magnetic fleld: $6.0 \mathrm{kG} ; 8.4 \mathrm{kG}$ and $10 \mathrm{kG}$. These fields are equivalent to kinetic energies of the accelerated beam of $4.2 \mathrm{GeV} ; 6.0 \mathrm{GeV}$ and $7.5 \mathrm{GeV}$.

The extracted beam was monitored with three scintillator detectors $S$, $\mathrm{S}_{2}$, and $\mathrm{S}_{3}$. Detector $\mathrm{S}_{1}$ (measuring $300 \mathrm{~mm}$ horizontally and $150 \mathrm{~mm}$ vertically) was positioned in the accelerator chamber and aligned with the direction of extraction. Readings from this detector were taken by a current integrator. Detector $\mathrm{S}_{2}$ (measuring $300 \times 300 \mathrm{~mm}^{2}$ ) was positioned in the head section of the extraction channel behind the first lens, and registered the extracted beam. Detector $\mathrm{S}_{3}$ (measuring $10 \times 100 \mathrm{~mm}^{2}$ ) was located in the experiment hall.

In the course of the experiment we investigated the dependence of the intensity of the beam counted by detectors $S_{1}, S_{2}$, and $s_{3}$ on the angular position of the bent silicon single crystal.

Figure 2 shows the orientation dependences for three positions of the crystal - at distances of 300,250 , and $200 \mathrm{~mm}$ from the center of the accelerator chamber at a beam energy $4.2 \mathrm{GeV}$.

It can be seen that when the crystal is closer to the central equilibrium orbit, the intensity of the beam at the location of detector $\mathrm{S}_{2}$ increases. This is explained by the fact that at a radial position of minus $300 \mathrm{~mm}$ (that is, further away from the central orbit) particles deflected by the crystal do not get into the exit branch of the channel (Fig. 2a). In all the figures, the orientation dependences are given for $10^{10}$ protons onto the targetdeflector.

Figure 3 shows orientational dependences at radial target position of minus $175 \mathrm{~mm}$ for two different energies of the accelerated beam -6.0 and 7.5 $\mathrm{GeV}$.

The widths of the orientation peaks are determined by the beam angular dispersion in the accelerator and, possibly, by the bent crystal surface deviation from cylindrical. The decrease in the width of the peaks that occurs as the energy increases can be explained by changes in the angular characteristics of the particle beam hitting the crystal, which may depend on the beam-aiming conditions.

Figure $3 a$ shows the dependences of the extracted beam intensity on the crystal orientation immediately after the exit from the accelerator chamber and in the experimental hall.

The shift in the orientation curves shows a discrepancy between the axis of the extracted beam and the axis of the channel head section. This, apparently, explains the substantial loss of intensity in the channel. These drawbacks can be eliminated by more accurate adjustment of the channel elements. 
The existing channel is designed for energies up to $6.0 \mathrm{GeV}$ maximum. This made it impossible to extract a beam with an energy of $7.5 \mathrm{GeV}$ to the experiment hall.

These results demonstrate the possibility of extracting accelerated particle beams by means of a bent crystal.

The effective beam extracted by the crystal deflector in this case was equal to $10^{-4}$ and was determined in general by the geometries of the beam and the crystal. This effectiveness can be substantially increased by increasing the transverse size of the crystal deflector.

In conclusion, the authors wish to thank Akademician A. M. Baldin, L. P. Zinov'yev, I. N. Semenyushkin, and A. D. Kirillov for their support and the interest shown in the course of the experiment.

\section{REFERENCES :}

1. Tsyganov, E. N. Fermilab TM-682, Batavia, 1976.

2. Elishev, A. F. et al. Phys. Lett. 88B, 387 (1979).

3. Bak, J. et al., Phys. Lett. 93B, 505 (1980).

4. Andreev, V. A. et al., Lentngrad Inst. of Nuclear Phys. Preprint \#792, 1982 .

5. Baker, S. I. et a1., Phys. Lett. 173B, 129 (1984).

6. Koshkarev, G. D., Inst. of Theor, and Experim. Phys. Preprint $\$ 30,1977$.

7. Carrigan, R. A. et a1., Nuc1. Instrum. Methods, 194, 205 (1982). 


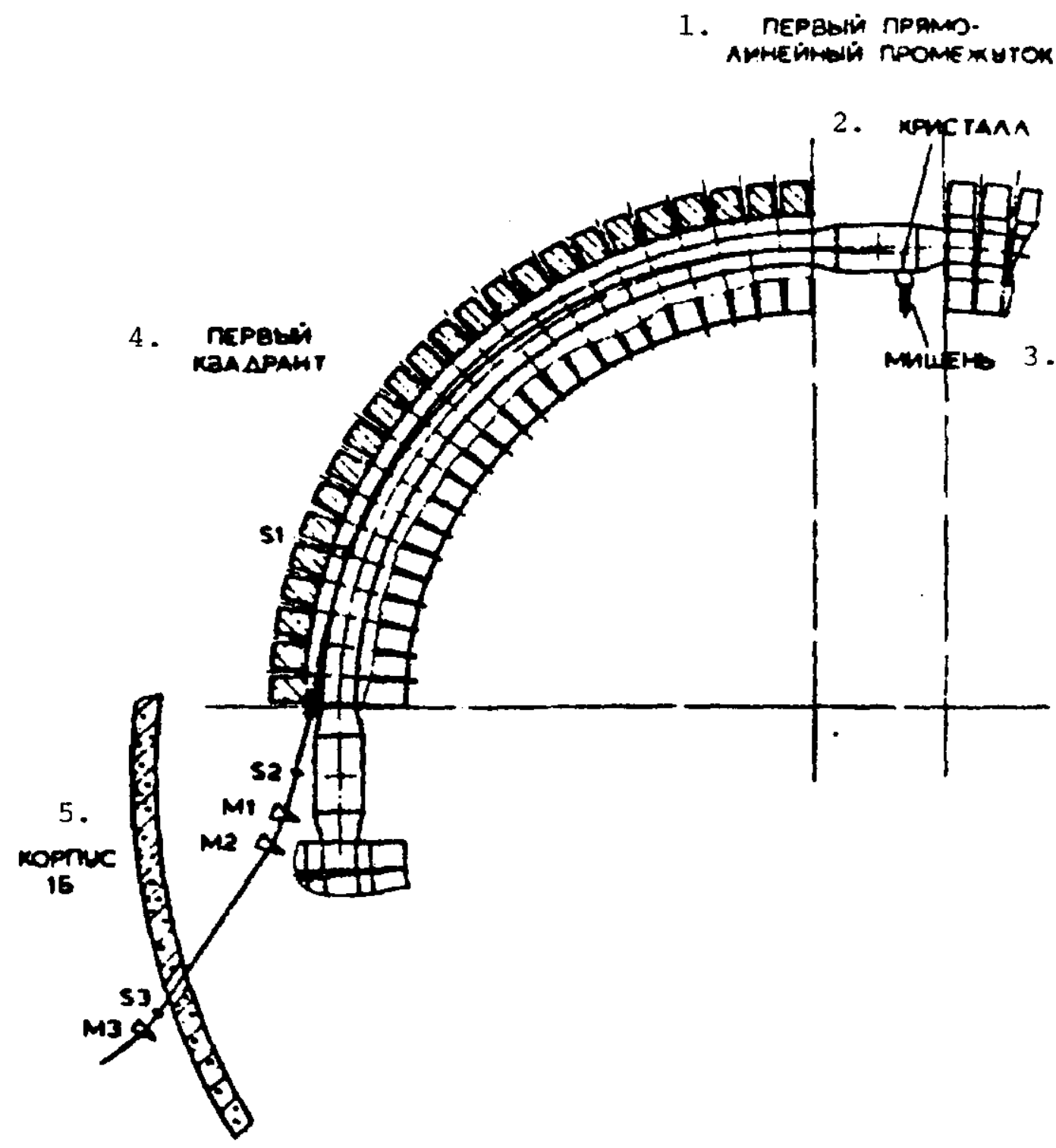

Fig. 1 shows the schematic layout of the experiment. 1- first straight section

2- crystal; 3- target; 4-first quadrant;

5- experimental hall 


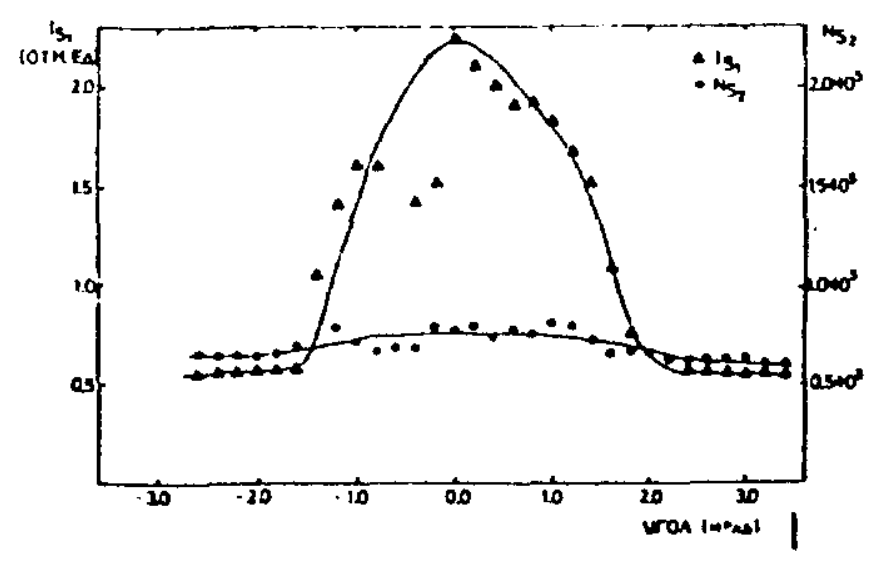

$2 a$

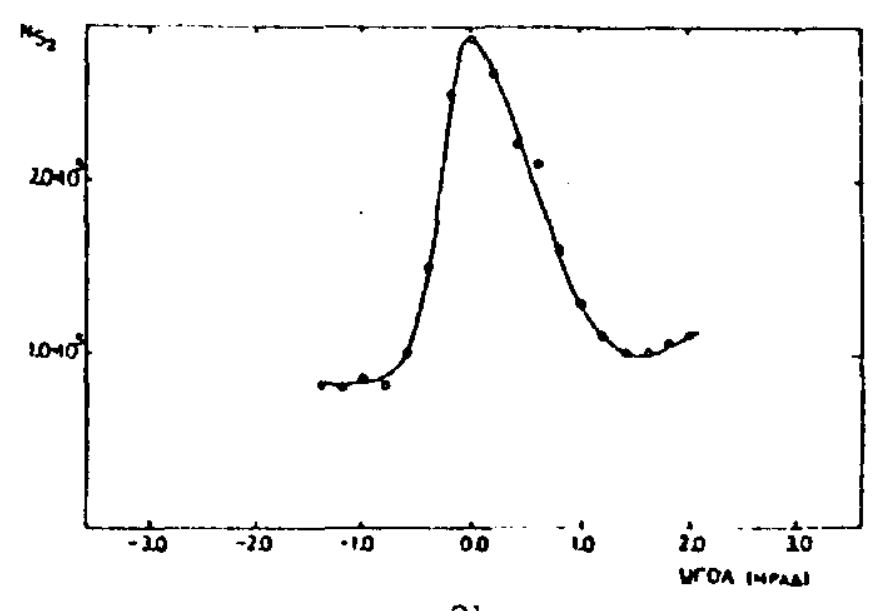

$2 b$

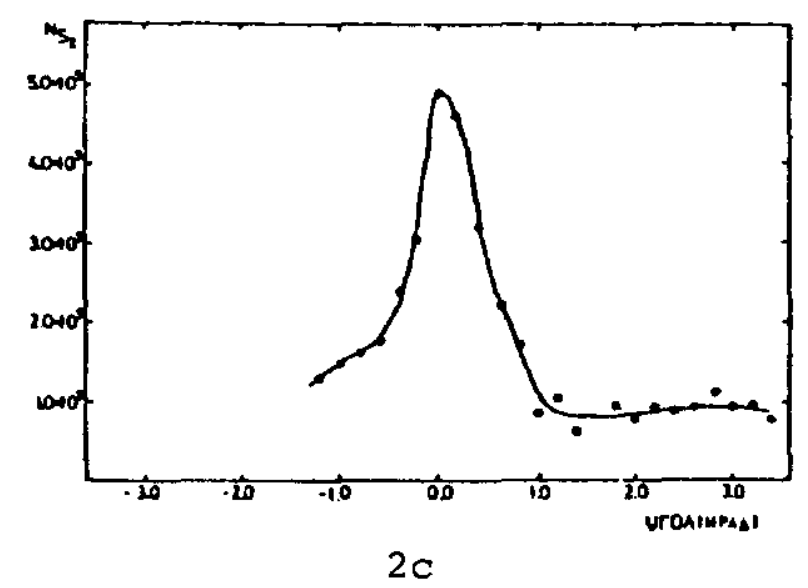

F1g. 2. Dependence of the extracted beam intensity on crystal orientation at 4.2 GeV for varfous radial positions of the crystal deflector: a) $-300 \mathrm{~mm} / \mathrm{I}_{\mathrm{S}_{1}}$ is the integrated current from detector $\mathrm{S}_{1} / ;$ b) $-250 \mathrm{~mm}$; c) $-200 \mathrm{~m}$. The ordinates represent $S$ singles rates per spill. On figure $2 a$ an integrated current per $s p i l 1$ in $S$ in arbitrary units is also shown on the left hand axis. The horlzontal axis is the angle between the crystal and the accelerator beam and ranges from -3 to +3 milliradians. The background is due to secondary radiation. The vertical axes in figures $b$ and $c$ are in units of $10^{5}$. 

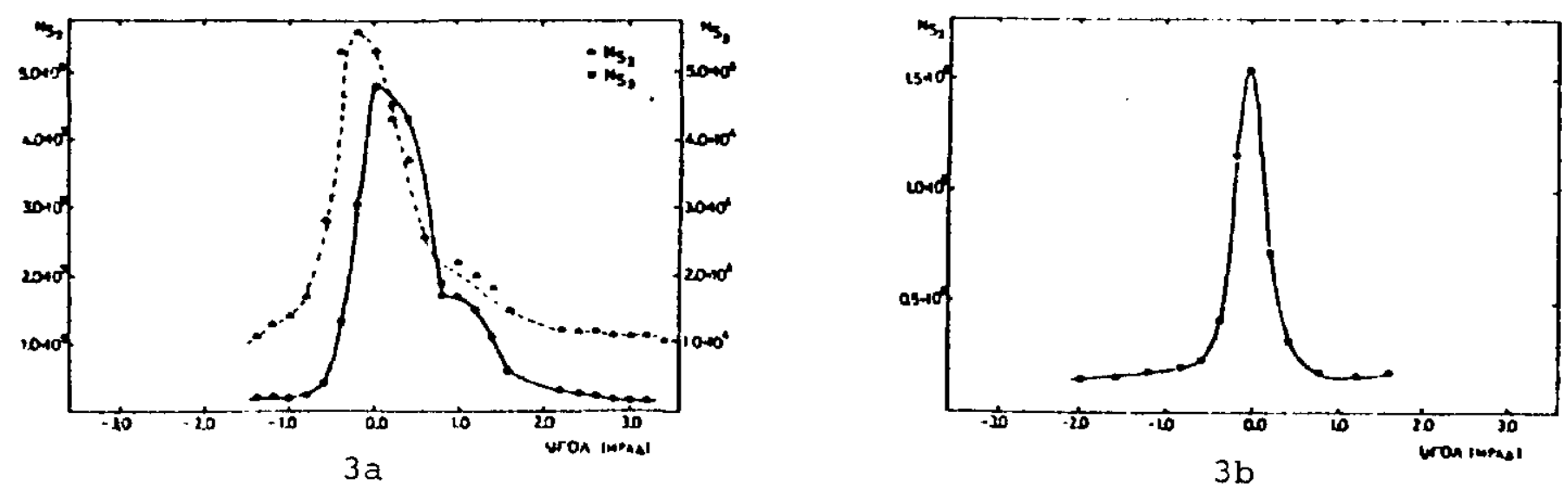

Fig. 3. Dependence of extracted beam intensity on crystal orientation for $6.0 \mathrm{GeV}$ and $7.5 \mathrm{GeV}$ at a crystal radial position of $-175 \mathrm{~mm}$. a) for an energy of $6.0 \mathrm{GeV}$; b) for an energy of $7.5 \mathrm{GeV}$. The horizontal axis is the same as Fig. 2. The background is due to secondary radiation. The left hand axes are $S_{2}$ in units of $10^{5}$. 